\title{
Discretization Error Based Mesh Generation for Diffuse Optical Tomography
}

\author{
Murat Guven, Birsen Yazici, Kiwoon Kwon \\ Electrical, Computer, and Systems \\ Engineering Department \\ Rensselaer Polytechnic Institute \\ Troy, NY, USA
}

\author{
Eldar Giladi \\ Department of Mathematical Sciences, \\ Rensselaer Polytechnic Institute, \\ Troy, NY, USA
}

\author{
Xavier Intes \\ ART Advanced Research Technologies, \\ Quebec, Canada
}

\begin{abstract}
In this paper, we analyze the perturbation in the reconstructed optical absorption images, resulting from the discretization of the forward and inverse problems. We show that the perturbation due to each problem is a function of both the forward and inverse problem solutions and can be reduced by proper refinement of the discretization mesh. Based on the perturbation analysis, we devise a novel adaptive discretization scheme for forward and inverse problems, which reduces the perturbation on the reconstructed image. Such a discretization scheme leads to an adaptively refined composite mesh sufficient to approximate the forward and inverse problem solutions within a desired level of accuracy while keeping the computational complexity within the computational power limits.
\end{abstract}

\section{Introduction}

Diffuse Optical Tomography (DOT) is a non-invasive imaging modality that makes use of the light in the NearInfrared (NIR) spectrum [1]. The inverse problem in DOT involves reconstruction of spatially varying absorption and scattering properties as well as fluorophore lifetime and yield in tissues from boundary measurements. These fundamental quantities can be utilized to obtain tissue oxy- and deoxyhemoglobin concentrations, blood oxygen saturation, water, fat, and contrast agent uptake in tissue [2], use of which find applications in breast cancer diagnosis [3], cognitive activity monitoring [4], brain tumor and hemorrhage detection [5], and in fluorescence tomographic imaging [6].

DOT imaging is comprised of two interdependent stages which seek solutions to the forward and the inverse problems. In this work, we analyze the effect of forward and inverse problem discretizations on the accuracy of the reconstructed optical absorption images. Based on this analysis, we propose a novel adaptive discretization scheme, in an attempt to directly reduce the perturbation in the reconstructed optical image, which arises due to the discretization of the coupled forward and inverse problems. We note that the discretization error in each problem does not directly induce a perturbation in the reconstructed image. We show that there are other factors which may spatially amplify or reduce the effect of the discretization error at each stage. As a result, the proposed discretization scheme takes the intrinsic properties of the imaging problem, such as the mutual dependence of forward and inverse problems, sourcedetector configuration and inverse problem formulation into account. Therefore, the proposed adaptive discretization approach outperforms the generic adaptive and uniform refinement schemes, while keeping the computational complexity to minimum.

So far, several investigators reported adaptive discretization based schemes for DOT imaging. We have presented a region-of-interest (ROI) imaging scheme for DOT[14], which employed a multi-level algorithm on a nonuniform grid. The non-uniform grid is designed so as to provide finer spatial resolution for the ROI which corresponds to the tumor region as indicated by the $a$ priori anatomical image. Torregrossa et al proposed an a priori non-uniform mesh design which provides high resolution for the heterogeneities as well as for the near-boundary regions [10]. Gu et al proposed a dual mesh strategy, in which case, a relatively finer uniform mesh is considered for the forward problem discretization and a coarser uniform mesh is generated for the inverse problem discretization [11]. In the same study, a dynamic adaptive scheme has been proposed for the inverse problem discretization as well, however no adaptive refinement is considered for the solution of the for- 
ward problem. Furthermore, the influence of the forward problem solution on the reconstructed image accuracy is not considered in the design of the adaptive mesh. Huang and Zhu (also presented a dual-mesh strategy that makes use of a priori ultrasound information [12]. In this work, the dual mesh implies a coarse mesh for background tissue and a relatively finer mesh for the heterogeneity, similar to the approach in [14]. Joshi et al used a 3D mesh to discretize the inverse fluorescence imaging problem and the associated coupled diffusion equations [13]. An adaptive finite element method is used for discretization where the refinement criterion is based on a posteriori interpolation error estimates. However, the mesh generation disregards the interdependence between the forward and inverse problems and ignores the dependence on the regularization parameter.

In this work, the forward problem is defined by the frequency-domain diffusion equation and the associated adjoint problem. As for the inverse problem, we consider the linear integral equation resulting from the Born approximation with a zero order Tikhonov regularizer. We use finite elements and linear Lagrange basis functions for approximating the forward and inverse problem solutions. Our perturbation analysis is confined to showing the impact of the discretization error in the forward and inverse problem solutions on the reconstructed optical absorption image. In this context, we disregard the perturbation due to other error sources such as model inconsistencies (due to the diffusion model, linearization of the inverse problem, and noise), numerical algorithm efficiency and inverse problem formulation. The perturbation analysis portrays the dependence of the image quality on the optical image properties, the configuration of source and detectors and the parameters involved in the inverse problem formulation, such as the regularization parameter. In our analysis, we show that contribution in the perturbation due to forward problem depends on the inverse problem solution, whereas the contribution due to inverse problem depends on the forward problem solution. Thus, discretization error in each problem may be amplified based on the spatial behavior of the solution of the other problem. We note that the discretization scheme described here can be either a priori, a posteriori or both, depending on the availability and/or reliability of a priori anatomical information.

\section{Forward and Inverse Problems}

In this section, we define the integral equation describing the inverse DOT problem and introduce the components of the inverse problem formulation with the associated notation as well as the underlying forward model.

We model the NIR light propagation in a bounded do- main $\Omega \subset R^{3}$ with the following boundary value problem:

$$
\begin{array}{r}
-\nabla \cdot D(\mathbf{x}) \nabla g+\left(\mu_{a}(\mathbf{x})+\frac{i \omega}{c}\right) g=\delta\left(\mathbf{x}-\mathbf{x}_{s}^{j}\right) \quad \mathbf{x} \in \Omega, \\
g+2 A D(\mathbf{x}) \frac{\partial g}{\partial n}=0 \quad \mathbf{x} \in \partial \Omega .
\end{array}
$$

where $\mathbf{x} \in R^{3}, D(\mathbf{x})$ is the spatially varying diffusion coefficient, $\mu_{a}(\mathbf{x})$ is the spatially varying absorption coefficient, $\omega$ is the modulation frequency of the point source $\delta\left(\mathbf{x}-\mathbf{x}_{s}^{j}\right)$ and $A=(1+R) /(1-R)$ where $R$ is a parameter governing the internal reflection at the boundary $\partial \Omega$.

Based on the Born approximation, the following linear integral equation relates the differential optical measurements to a small perturbation $\alpha(\mathbf{x})$ on the absorption coefficient $\mu_{a}(\mathbf{x})$ :

$$
\begin{aligned}
\Gamma\left(\mathbf{x}_{d}^{i} ; \mathbf{x}_{s}^{j}\right) & =-\int_{\Omega} \overline{g^{*}\left(\dot{\mathbf{x}} ; \mathbf{x}_{d}^{i}\right)} g\left(\dot{\mathbf{x}} ; \mathbf{x}_{s}^{j}\right) \alpha(\dot{\mathbf{x}}) d \dot{\mathbf{x}} \\
& =\int_{\Omega} H\left(\dot{\mathbf{x}} ; \mathbf{x}_{d}^{i}, \mathbf{x}_{s}^{j}\right) \alpha(\dot{\mathbf{x}}) d \dot{\mathbf{x}} \\
& =A_{a} \alpha,
\end{aligned}
$$

where $\left(\mathbf{x}_{d}^{i}\right), i=1, \ldots, N_{d}$ and $\left(\mathbf{x}_{s}^{j}\right), j=1, \ldots, N_{s}$ stand for the detector and source positions, respectively and $\Gamma\left(\mathbf{x}_{d}^{i} ; \mathbf{x}_{s}^{j}\right)$ represents the perturbation in the measurement at the $j^{t h}$ detector due to the $i^{t h}$ source and $\Omega \subset R^{3}$. $H\left(\hat{\mathbf{x}} ; \mathbf{x}_{d}^{i}, \mathbf{x}_{s}^{j}\right)=-\overline{g^{*}\left(\hat{\mathbf{x}} ; \mathbf{x}_{d}^{i}\right)} g\left(\hat{\mathbf{x}} ; \mathbf{x}_{s}^{j}\right)$ is the kernel of the integral operator $A_{a}: L^{\infty} \rightarrow C^{N_{d} \times N_{s}}$. In $3, g^{*}$ is the solution to the adjoint problem [9]:

$$
\begin{array}{r}
-\nabla \cdot D(\mathbf{x}) \nabla g^{*}+\left(\mu_{a}(\mathbf{x})-\frac{i \omega}{c}\right) g^{*}=0 \quad \mathbf{x} \in \Omega \\
g^{*}+2 A D(\mathbf{x}) \frac{\partial g^{*}}{\partial n}=\delta\left(\mathbf{x}-\mathbf{x}_{d}^{i}\right) \quad \mathbf{x} \in \partial \Omega, .
\end{array}
$$

In this work, we consider Tikhonov regularization for our illustrative purposes, which yields the following equation in terms of $\alpha^{\lambda}$ which approximates $\alpha$ :

$$
\begin{aligned}
\gamma=A_{a}^{*} \Gamma_{i j} & =\left(A_{a}^{*} A_{a}+\lambda I\right) \alpha^{\lambda} \\
& =K \alpha^{\lambda}
\end{aligned}
$$

for some $\lambda>0$. In this representation $A_{a}^{*}: C^{N_{d} \times N_{s}} \rightarrow L^{1}$ is the adjoint of $A_{a}$ and $I$ is the identity operator. Let $A=$ $A_{a}^{*} A_{a}$, then $A: L^{\infty} \rightarrow L^{1}$ is defined as follows:

$$
\begin{gathered}
(A \alpha)(\mathbf{x})=\sum_{i, j}^{N_{d}, N_{s}} H^{*}\left(\mathbf{x} ; \mathbf{x}_{d}^{i}, \mathbf{x}_{s}^{j}\right) \int_{\Omega} H\left(\mathbf{x}_{d}^{i}, \mathbf{x}_{s}^{j} ; \dot{\mathbf{x}}\right) \alpha(\dot{\mathbf{x}}) d \dot{\mathbf{x}}, \\
=\int_{\Omega} \kappa(\mathbf{x} ; \dot{\mathbf{x}}) \alpha(\dot{\mathbf{x}}) d \dot{\mathbf{x}},
\end{gathered}
$$

where $\kappa(\mathbf{x} ; \dot{\mathbf{x}})$ stands for the kernel of the integral operator $A$ and is given by

$$
\kappa(\mathbf{x} ; \dot{\mathbf{x}})=\sum_{i, j}^{N_{d}, N_{s}} H^{*}\left(\mathbf{x} ; \mathbf{x}_{d}^{i}, \mathbf{x}_{s}^{j}\right) H\left(\dot{\mathbf{x}} ; \mathbf{x}_{d}^{i}, \mathbf{x}_{s}^{j}\right),
$$


where we derive the kernel $H^{*}\left(\mathbf{x} ; \mathbf{x}_{d}^{i}, \mathbf{x}_{s}^{j}\right)$ of the adjoint operator in the following section. Note that, in this work we assume that the solution $\alpha^{\lambda} \in L^{\infty}$ also satisfies $\alpha^{\lambda} \in H^{1}$.

From this point on, we will replace $L^{\infty}$ and $L^{1}$ with $X$ and $Y$, respectively, where necessary for notational convenience. Thus $K: X \rightarrow Y$. Note also that $X \subset Y$.

\subsection{Operator Approximation and Dis- cretization of the Inverse Problem}

In practice, we seek a finite-dimensional approximation to the solution of the inverse problem defined by equation 8 . Therefore, we discretize the inverse problem defined by equation 8 by projecting it onto a finite dimensional subspace $X_{n} \subset X$.

Let $X_{n}$ be a sequence of finite-dimensional subspaces of dimension $n$, spanned by the linear Lagrange basis functions $\left\{L_{1}, \ldots, L_{n}\right\}$ and $\left\{\mathbf{x}_{k}\right\}$ for $k=1, \ldots, n$ be the set of projection points. Then the projection operator $P_{n}: X \rightarrow$ $X_{n}$ for collocation method is defined by

$$
P_{n} f(\mathbf{x})=\sum_{k=1}^{n} f\left(\mathbf{x}_{k}\right) L_{k}(\mathbf{x}), \quad(\mathbf{x}) \in \Omega,
$$

for all $f(\mathbf{x}) \in X$.

Projecting equation 8 yields:

$$
P_{n} K \alpha_{n}^{\lambda}=P_{n} \gamma
$$

The operator $P_{n} K=\left(k_{l k}\right) \in C^{n \times n}$ is a complex matrix where $n$ is the size of the matrix. Applying projection by collocation [8], the projection for the operator $K$ yields

$\lambda a_{j}+\sum_{k=1}^{n} a_{k} \int_{\Omega} \kappa\left(\mathbf{x}_{j} ; \dot{\mathbf{x}}\right) L_{k}(\dot{\mathbf{x}}) d \dot{\mathbf{x}}=\gamma\left(\mathbf{x}_{j}\right), j=1, \ldots, n$,

where we expressed $\alpha_{n}^{\lambda}$ in terms of the linear Lagrange basis functions $L_{k}$ as

$$
\alpha_{n}^{\lambda}=\sum_{k=1}^{n} a_{k} L_{k}
$$

Therefore $k_{l k}$ is given by

$$
k_{l k}=\lambda \delta_{l k}+\int_{\Omega} \kappa\left(\mathbf{x}_{l} ; \dot{\mathbf{x}}\right) L_{k}(\dot{\mathbf{x}}) d \dot{\mathbf{x}}
$$

for $l=1, \ldots, n$, and $k=1, \ldots, n$, where $\delta_{l k}=0$ unless $l=k$. However, projection methods are only semi-discrete, thus equation 11 does not provide a fully discrete model. Furthermore, in practice, we only have approximations to the operator $K$ and the right hand side $\gamma$. Using a finite dimensional approximation for the kernel $\kappa(\mathbf{x})$, we have a full discrete model for the approximating sequence $\tilde{\alpha}_{n}^{\lambda}$, given by the following relationship:

$$
P_{n} K_{n} \tilde{\alpha}_{n}^{\lambda}=P_{n} \tilde{\gamma}_{n}
$$

In equation 13, the operator $K_{n}: X \rightarrow Y$ is a finite dimensional approximation of the original operator $K$ and $P_{n} K_{n}: X_{n} \rightarrow Y_{n}$. Similarly

$$
\tilde{\gamma}_{n}=A_{a n}^{*} \Gamma
$$

where $A_{a n}^{*}$ is the approximation to the adjoint operator $A_{a}^{*}$. Note that $K_{n} \rightarrow K$ and $\tilde{\gamma}_{n} \rightarrow \gamma$ as $n \rightarrow \infty$.

As a result of operator approximation and discretization of the inverse problem, the reconstructed image $\tilde{\alpha}_{n}^{\lambda}$ is merely an approximation to the actual image $\alpha^{\lambda}$. The outcome of projecting the inverse problem onto a finitedimensional sub-space and the discretization error in the solution of the forward problem is perturbation in the reconstructed image. Therefore, the accuracy of the reconstructed image is challenged by the discretization followed in forward and inverse problem solutions.

We analyze the error in the solution $\tilde{\alpha}_{n}^{\lambda}$ of equation 13 with respect to the actual solution $\alpha^{\lambda}$ of equation 8 , in two steps. We write $\tilde{\alpha}_{n}^{\lambda}=\tilde{\alpha}^{\lambda}-e_{n}$, where $e_{n}$ is the error resulting from only projection of the inverse problem with the degenerate kernel and denote $\tilde{\alpha}^{\lambda}=\alpha^{\lambda}-\tilde{e}$, where $\tilde{e}$ is the error due to operator kernel approximation. As a result, we arrive at the following straightforward conclusion:

$$
\tilde{\alpha}_{n}^{\lambda}=\tilde{\alpha}^{\lambda}-e_{n}=\alpha^{\lambda}-e_{n}-\tilde{e} .
$$

Therefore, we can write an upper bound on the perturbation $\alpha^{\lambda}-\tilde{\alpha}_{n}^{\lambda}$ as follows:

$\left\|\alpha^{\lambda}-\tilde{\alpha}_{n}^{\lambda}\right\|=\left\|\alpha^{\lambda}-\tilde{\alpha}^{\lambda}+\tilde{\alpha}^{\lambda}-\tilde{\alpha}_{n}^{\lambda}\right\| \leq\|\tilde{e}\|+\left\|e_{n}\right\|$.

In the following section, we outline a different perspective where we explore the effect of projection and forward problem discretization separately.

\section{Discretization-based Error Analysis}

In this section, we discuss how discretization of forward and inverse problems impacts the accuracy of the DOT imaging. The analysis is carried out based on the inverse problem defined by equation 8 and the associated kernel $\kappa(\mathbf{x} ; \mathbf{\mathbf { x }})$. We first consider the impact of projection (i.e. inverse problem discretization) by collocation method when the kernel of the integral operator is exact, or equivalently, when there is no discretization error in the forward problem solution. Next, we explore the practical case where the kernel is replaced by its finite-dimensional approximation and interpret how discretization error in the forward problem solution impacts the optical imaging accuracy. 
The two investigations reveal that even if there is no discretization error in the kernel approximation, the accuracy of the solution approximation $\tilde{\alpha}_{n}^{\lambda}$ depends on the kernel $\kappa(\mathbf{x} ; \dot{\mathbf{x}})$ of the integral operator. Furthermore, the perturbation in the reconstructed optical image due to discretization error in the forward problem solution is a function of the inverse problem solution. These results suggest that the discretization of the inverse and forward problems can not be considered independent of each other.

\subsection{Case 1: The kernel is exact}

Theorem 1 Let $\left\{\Omega_{m}\right\}$ denote a set of triangular finite elements for $m=1, \cdots, N_{\Delta}$, such that $N_{\Delta} \Omega_{m}=\Omega$ and $h_{m}$ be the longest edge of the $m^{\text {th }}$ triangle. Then $\left\|\alpha^{\lambda}-\alpha_{n}^{\lambda}\right\|_{L^{1}(\Omega)} \leq$

$$
\begin{array}{r}
C A_{\Omega}\left\|\left(I-T_{n}\right)\right\|_{Y \rightarrow X_{n}} \sum_{m=1}^{N_{\Delta}}\left\|\alpha^{\lambda}\right\|_{1, m} h_{m} \\
+\frac{C}{\lambda}\left\|T_{n}\right\|_{Y \rightarrow X_{n}} \max _{i, j}\left\|g_{i}^{*}(\mathbf{x}) g_{j}(\mathbf{x})\right\|_{L^{1}(\Omega)} \\
\times \sum_{m=1}^{N_{\Delta}} \sum_{i, j}^{N_{d}, N_{s}}\left\|g_{i}^{*}(\dot{\mathbf{x}}) g_{j}(\dot{\mathbf{x}})\right\|_{0, m}\left\|\alpha^{\lambda}\right\|_{1, m} h_{m},
\end{array}
$$

where $T_{n}=\left[I+\frac{1}{\lambda} P_{n} A\right]^{-1} P_{n}: Y \rightarrow X_{n}$ is a uniformly bounded operator [15].

\subsection{Case 2: The kernel is degenerate}

Theorem 2 The operator approximation $K_{n}$ results in the perturbation in the reconstructed optical image. As a result, $\left\|\alpha^{\lambda}-\tilde{\alpha}^{\lambda}\right\|_{L^{1}(\Omega)} \leq$

$$
\begin{array}{r}
\frac{2 C / \lambda}{1-\left\|K^{-1}\left(K_{n}-K\right)\right\|} \\
\times \max _{i, j}\left\|g_{i}^{*}(\mathbf{x}) g_{j}(\mathbf{x})\right\|_{L^{1}(\Omega)}\left(\sum_{m=1}^{N_{\Delta}} \sum_{i, j}^{N_{d}, N_{s}}\right. \\
+\left(\left\|g_{j} \alpha^{\lambda}\right\|_{0, m}+\|\alpha\|_{0}\left\|g_{j}\right\|_{0, m}\right)\left\|g_{i}^{*}\right\|_{1, m} h_{m}^{i} \\
\left.+\left(\left\|g_{i}^{*} \alpha^{\lambda}\right\|_{0, m}+\|\alpha\|_{0}\left\|g_{i}^{*}\right\|_{0, m}\right)\left\|g_{j}\right\|_{1, m} h_{m}^{j}\right),
\end{array}
$$

where $h_{m}^{i}$ and $h_{m}^{j}$ are the longest edges of the $m^{t h}$ finite element in the solutions $g_{i}^{*}$ and $g_{j}$, respectively. Thus, we consider different meshes for the solutions $g_{i}^{*}$ and $g_{j}$ for $i=1, \ldots, N_{d}$ and $j=1, \ldots, N_{s}[15]$.

\subsection{Adaptive mesh generation}

In this section, we first discuss the adaptive mesh design for the discretization of forward and inverse problems, such that the resulting perturbation in the reconstructed optical image due to each discretization is below the allowable bound.

\subsubsection{Forward Problem Discretization}

Let the mesh parameter $h_{m}^{i}$ for $g_{i}^{*}$ and the mesh parameter $h_{m}^{j}$ for $g_{j}$ be adjusted as follows:

$$
\begin{aligned}
h_{m}^{i} & \leq \frac{\epsilon_{f}}{\sum_{j}^{N_{s}}\left(\left\|g_{j} \alpha^{\lambda}\right\|_{0, m}+\|\alpha\|_{0}\left\|g_{j}\right\|_{0, m}\right)\left\|g_{i}^{*}\right\|_{1, m}} \\
h_{m}^{j} & \leq \frac{\epsilon_{f}}{\sum_{i}^{N_{d}}\left(\left\|g_{i}^{*} \alpha^{\lambda}\right\|_{0, m}+\|\alpha\|_{0}\left\|g_{i}^{*}\right\|_{0, m}\right)\left\|g_{j}\right\|_{1, m}},
\end{aligned}
$$

where the tolerance $\epsilon_{f}$ will be defined later. By theorem 3, the perturbation in the reconstructed image due to forward problem discretization is bounded by:

$$
\begin{aligned}
\frac{2 C / \lambda}{1-\left\|K^{-1}\left(K_{n}-K\right)\right\|} & \max _{i, j}\left\|g_{i}^{*}(\mathbf{x}) g_{j}(\mathbf{x})\right\|_{L^{1}(\Omega)} \\
\times & N_{\Delta}\left(N_{d}+N_{s}\right) \epsilon_{f}=\tilde{\epsilon}^{f},
\end{aligned}
$$

where $\tilde{\epsilon}^{f}$ is the total allowable perturbation due to forward problem discretization and 19 implies the following value for $\epsilon_{f}$ :

$$
\epsilon_{f}=\frac{\lambda \tilde{\epsilon}^{f}}{2 C\left(N_{d}+N_{s}\right) N_{\Delta}} \frac{1-\left\|K^{-1}\left(K_{n}-K\right)\right\|}{\max _{i, j}\left\|g_{i}^{*}(\mathbf{x}) g_{j}(\mathbf{x})\right\|_{L^{1}(\Omega)}} .
$$

\subsubsection{Inverse Problem Discretization}

Similarly we define the mesh parameter $h_{m}$ for the inverse problem discretization as follows:

$$
\begin{array}{r}
h_{m}=\epsilon_{i n v} /\left(C A_{\Omega}\left\|\left(I-T_{n}\right)\right\|_{Y \rightarrow X_{n}}\left\|\alpha^{\lambda}\right\|_{1, m}\right. \\
+\frac{1}{\lambda}\left\|T_{n}\right\|_{Y \rightarrow X_{n}} \max _{i, j}\left\|g_{i}^{*}(\mathbf{x}) g_{j}(\mathbf{x})\right\|_{L^{1}(\Omega)} \\
\left.\quad \times \sum_{i, j}^{N_{d}, N_{s}}\left\|g_{i}^{*}(\mathbf{x}) g_{j}(\dot{\mathbf{x}})\right\|_{0, m}\left\|\alpha^{\lambda}\right\|_{1, m}\right),
\end{array}
$$

for

$$
N_{\Delta} \epsilon_{i n v}=\tilde{\epsilon}^{i n v},
$$

where $\tilde{\epsilon}^{i n v}$ is the total allowable perturbation due to inverse problem discretization.

\section{Adaptive Mesh Refinement}

We consider a 2D rectangular domain $\Omega \subset R^{2}$ and generate an initial uniform triangular-element mesh which consists of right triangles. Based on the theorems 1,2 and the described adaptive mesh generation scheme, we apply $\mathrm{Ri}$ vara's algorithm [7] to adaptively refine the specified elements in forward and inverse problem discretizations. 


\section{Results}

We consider a simple case to show the effectiveness of the proposed discretization scheme.

Figure ?? shows a small heterogeneity close to the source side. The domain $\Omega$ is a square with a side of $6 \mathrm{~cm}$. There are 11 sources on the side closer to the heterogeneity and 11 detectors on the other side. We consider two cases: In the first case, we use a uniform mesh with 361 nodes for the solutions of the forward and inverse problems (figure 2). In the second case, we generate an adaptive mesh for both the forward and inverse problems as described in the previous section. We show the adaptive mesh with 339 nodes, generated for the inverse problem in figure 3 . The reconstructed images are shown in figures 4 and 5 , corresponding to the uniform and adaptive meshes, respectively. We observe the improved quality in figure 5, where we used an adaptive mesh for the discretization.

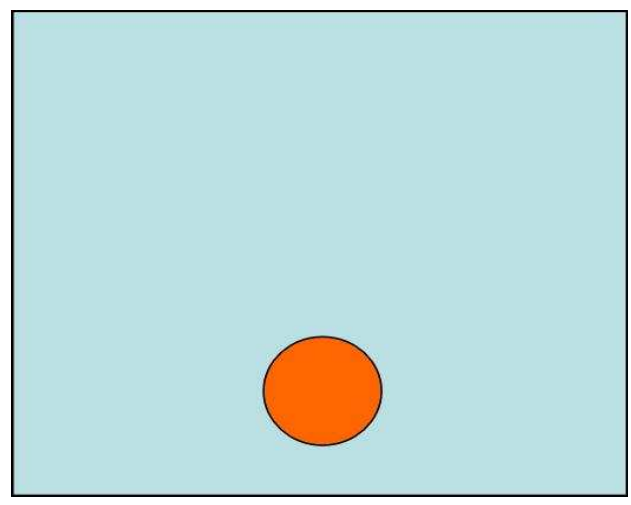

Figure 1. The optical medium used in the simulation experiment. The circular inclusion corresponds to the perturbation in the absorption coefficient of the medium

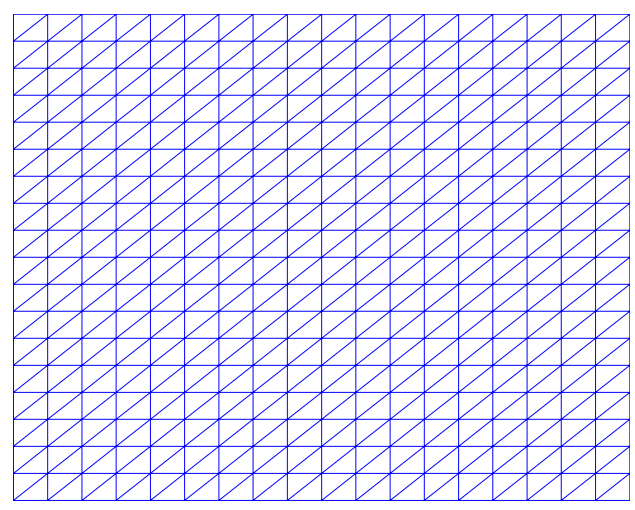

Figure 2. The uniform mesh.

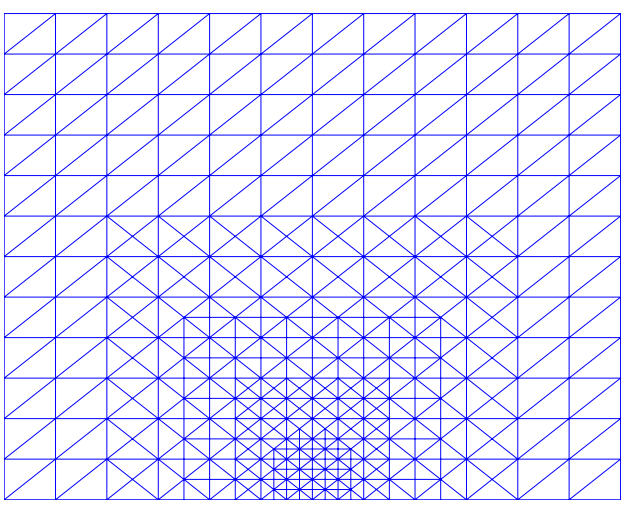

Figure 3. The adaptive mesh.

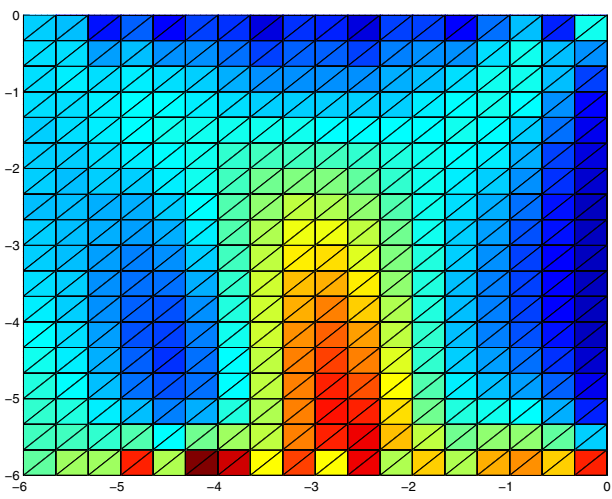

Figure 4. The reconstructed image where we used the uniform mesh for discretization.

\section{Conclusion and Future Work}

In this work, we proposed a novel adaptive discretization approach for diffuse optical tomography. We showed the effectiveness of the proposed adaptive discretization scheme on a $2 \mathrm{D}$ problem. The proposed discretization scheme enhances the image quality while keeping the computational complexity to minimum.

The perturbation analysis presented in this paper is valid for the 3D case as well. The computational savings in 3D will be much more than in 2D. Therefore, as for the future work, we will show the computational benefits of the proposed adaptive discretization scheme on 3D examples.

\section{References}

[1] Intes X and Chance B 2005 Non-PET functional imaging techniques: optical, The Radiologic Clinics of North America 43(1) 221-34. 


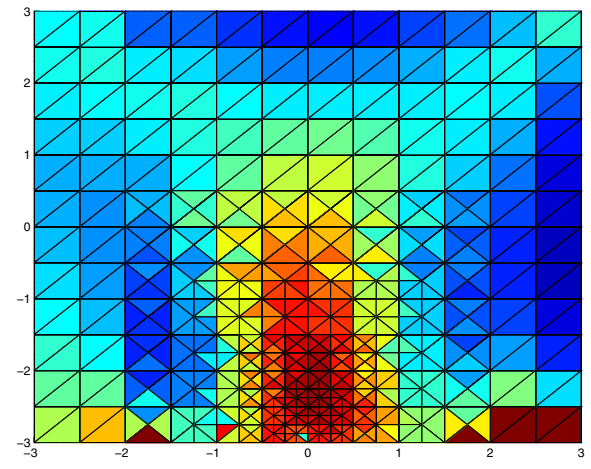

Figure 5. The reconstructed image where we used the adaptive mesh for discretization.

[2] Kincade K 2004 Optical Diagnostics continue migration from bench top to beside Laser Focus World 130-4.

[3] Intes X, Ripoll J, Chen Y, Nioka S, Yodh A and Chance B 2003 In vivo continuous-wave optical breast imaging enhanced with Indocyanine Green Med. Phys. Biol. 30, 1039-47.

[4] Strangman G, Boas D and Sutton J 2002 Non-invasive neuroimaging using near-infrared light Biol. Psychiatry 52 679-93.

[5] Hebden J C, Gibson A, Austin T, Yusof R, Everdell N, Delpy E, Arridge S R, Meek J H and Wyatt J S 2004 Imaging changes in blood volume and oxygenation in the newborn infant brain using three-dimensional optical tomography Phys. Med. Biol.49 1117-30.

[6] Weissleder R and Ntziachristos V 2003 Shedding light onto live molecular targets Nature Medicine 9 123-8.

[7] Rivara M C, 1984 "Mesh Refinement Processes Based on the Generalized Bisection of Simplices," SIAM J. Numer. Anal. 21 (3).

[8] Kress R, Linear Integral Equations, 1999, Springer Verlag.

[9] Arridge S R, "Optical Tomography in Medical Imaging," 1999, Inverse Problems 15, R41-R93.

[10] Torregrossa M, Zint C V, Poulet P, "Image reconstruction in optical tomography: mesh influence," 2002, IV International Workshop "Computational Problems of Electrical Engineering”, 183-6.

[11] Gu X, Xu, Y, Jiang H, "Mesh-based enhancement schemes in diffuse optical tomography," 2003, Med. Phys. 30 (5) 861-9.
[12] Huang M and Zhu Q, "Dual-mesh optical tomography reconstruction method with a depth correction that uses a priori ultrasound information," 2004 Applied Optics 43, no $8,1654-62$.

[13] Joshi A, Bangerth W, Sevick-Muraca E M, "Adaptive finite element based tomography for fluorescence optical imaging in tissue," 2004 Optics Express 12, no 22, 540217.

[14] Guven M, Intes X, Yazici B and Chance B, "An Adaptive Multigrid Algorithm for Region of Interest Diffuse Optical Tomography," 2003, Proceedings of International Conference on Image Processing, Volume 2, 823-6.

[15] Guven M, Kwon K, Yazici B, Giladi E and Intes X, "Discretization-error Based Mesh Generation for Diffuse Optical Tomography,'(in preparation). 